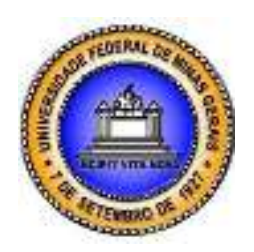

\title{
QUAL O CUSTO DOS PROCEDIMENTOS CIRÚRGICOS ESTÉTICOS MAIS PROCURADOS PELOS BRASILEIROS?
}

\section{WHAT IS THE COST OF AESTHETIC SURGICAL PROCEDURES MOST SOUGHT BY THE BRAZILIANS?}

\section{¿CUÁL ES EL COSTE DE LOS PROCEDIMIENTOS QUIRÚRGICOS ESTÉTICOS MÁS SOLICITADOS POR LOS BRASILEÑOS?}

Katia Abbas

Universidade Estadual de Maringá

kabbas@uem.br

Claudinéia Nunes Veloso

Universidade Estadual de Maringá

claudineia_triplice@hotmail.com

Joyce Menezes da Fonseca Tonin

Universidade Estadual de Maringá

joycemftonin@gmail.com
Submetido em: 20/03/2014

Artigo aceito em: 15/10/2014

\begin{abstract}
Resumo
A busca pela beleza coloca o Brasil em uma posição de destaque em número de cirurgias plásticas, ficando atrás apenas dos Estados Unidos. Os procedimentos cirúrgicos estéticos mais procurados pelos brasileiros são a lipoaspiração, a mamoplastia de aumento e a abdominoplastia. Assim, tendo em vista a importância da beleza para a sociedade, a escassez de pesquisas relacionadas ao custo dos procedimentos estéticos, e também a importância da obtenção do custo por procedimento, o objetivo deste trabalho é calcular o custo dos três procedimentos cirúrgicos estéticos mais procurados pelos brasileiros. Para tanto, baseado nas metodologias de Matos (2002) e Leoncine, Bornia e Abbas (2012), foram alocados aos procedimentos todos os insumos utilizados no fluxo percorrido pelo paciente nos serviços prestados pelo hospital, ou seja, tanto os custos diretos quanto os indiretos advindos da farmácia, do almoxarifado e dos centros de custos que foram obtidos por meio do método RKW. Constatou-se que não há diferenças significativas em relação aos custos dos materiais, medicamentos e dos centros produtivos para os procedimentos. No entanto, o procedimento cirúrgico mamoplastia, apresentou um custo maior em relação à lipoaspiração e à abdominoplastia, devido ao alto custo da prótese mamária.
\end{abstract}

Palavras-chave: Procedimentos Cirúrgicos Estéticos. Custo por Procedimento. Cirurgias Plásticas.

\begin{abstract}
The quest for beauty puts Brazil in a prominent position in number of plastic surgeries, trailing only the United States. The most sought after by Brazilians aesthetic surgical procedures are liposuction, breast augmentation and abdominoplasty. Thus, in view of the importance of beauty to society, the scarcity of research on the cost of cosmetic procedures, and also the importance of getting the cost per procedure, the objective of this work is to calculate the cost of the three most popular aesthetic surgical procedures by Brazilians. To do so, based on methodologies Matos (2002 ) and Leoncine, Bornia and Abbas (2012 ), all
\end{abstract}



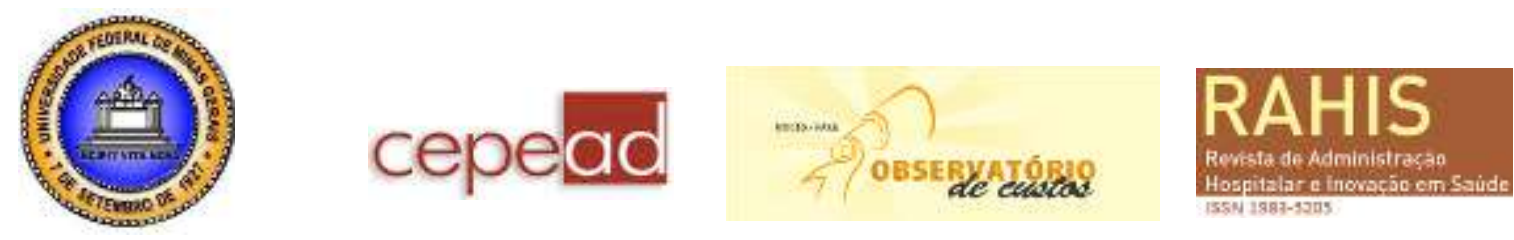

procedures were allocated to the inputs used in the flow driven by the patient in the services provided by the hospital, ie, both direct costs as indirect arising pharmacy, warehouse and cost centers that were obtained by the method RKW. It found no significant differences in the costs of materials, medicines and productive centers for procedures. However, breast augmentation surgery, showed a greater cost compared to liposuction and abdominoplasty, due to the high cost of breast implants.

Keywords: Surgical Aesthetic Procedures. Cost per procedure. Plastic Surgeries.

\section{Resumen}

La búsqueda de la belleza coloca a Brasil en una posición destacada en el número de cirugías plásticas, sólo por detrás de los Estados Unidos. Los más buscados por los brasileños procedimientos quirúrgicos estéticos son la liposucción, aumento de senos y cirugía estética de abdomen. Por lo tanto, en vista de la importancia de la belleza para la sociedad, la escasez de investigación sobre el costo de los procedimientos quirúrgicos estéticos, y también la importancia de obtener el coste por proceso, el objetivo de este trabajo es calcular el coste de las tres de la cirugía estética más popular por los brasileños. Para ello, sobre la base de metodologías Matos (2002) y Leoncine , Bornia y Abbas (2012), todos los procedimientos fueron asignados a los insumos utilizados en el flujo impulsado por el paciente en los servicios prestados por el hospital, es decir, tanto los costes directos como los indirectos derivada centros farmacia, trastero y de costos que se obtuvieron por el método de RKW. Se encontró que no hay diferencias significativas en los costos de materiales, medicamentos y centros productivos para los procedimientos. Sin embargo, la cirugía de levantamiento de senos, mostró un mayor costo en comparación con la liposucción y la abdominoplastia, debido al alto costo de los implantes mamarios.

Palabras clave: Procedimientos Quirúrgicos Estéticos. Tarifa por Procedimiento. Las Cirugías Plásticas.

\section{Introdução}

A busca pela beleza ou pela perfeição coloca o Brasil em uma posição de destaque em número de cirurgias plásticas, ficando atrás apenas dos Estados Unidos. Segundo dados da International Society of Aesthetic Plastic Surgeons (ISAPS), em 2011 foram realizados 905.124 procedimentos cirúrgicos estéticos, sendo que os mais procurados são a lipoaspiração (remoção da gordura localizada) que ocupa o primeiro lugar do ranking, seguido da mamoplastia de aumento (inserção de prótese para aumento da mama) que aparece em segundo lugar, e em terceiro lugar vem a abdominoplastia (remoção do excesso de gordura e de flacidez do abdômen) (ISAPS, 2013).

Esses dados, que refletem a insatisfação com a imagem e a consequente busca pela perfeição, são reforçados pela mídia, mais especificamente pela propaganda que apresenta pessoas com corpos e rostos perfeitos (FERRAZ; SERRALTA, 2007; FARIA, 2010).

Apesar deste número significativo de cirurgias, Aquino (2009) ressalta que a maior parte dos estudos sobre cirurgias plásticas se concentram na área médica, abordando a modernização de técnicas cirúrgicas e dos procedimentos de intervenção.

Há também alguns estudos que retratam as características das pessoas que se submetem a cirurgias plásticas estéticas, para verificar os fatores da propensão a submeter-se ao tratamento cirúrgico com finalidade estética (ASKEEGARD; GERTSEN; LANGER, 2002; AVELAR, 2011). 

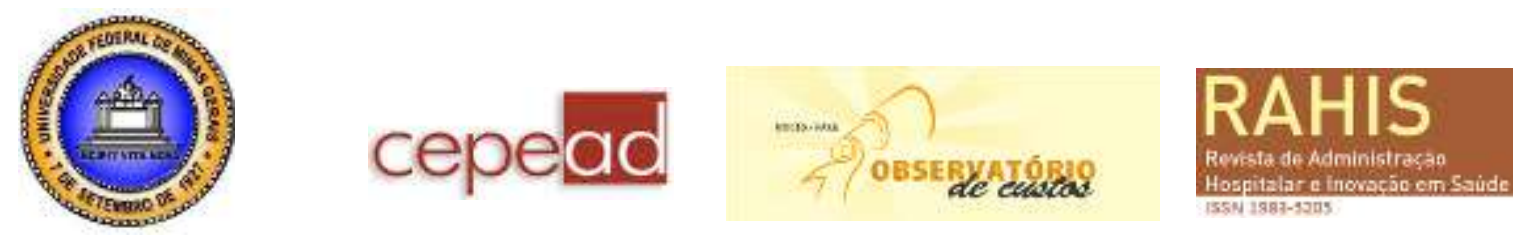

Em relação aos custos, muitas das pesquisas na área da saúde, tem se concentrado na aplicação dos métodos de custeio, mais especificamente o custeio baseado em atividade e o método das seções homogêneas, para o cálculo dos custos de centros de custos, bem como de atividades (GOULART, 2000; ABBAS, 2001; MARTINS, 2002; VARGAS, 2002; NERI, 2006; SCHULTZ, 2007; SOUZA et al. 2008, SOUZA et al. 2012).

Leoncine (2010, p. 132) em seu trabalho propõe uma sistemática para apuração dos custos por procedimento médico, e o mesmo ressalta que "o custo por procedimento médico hospitalar fornece informação estratégica para a tomada de decisão".

Leoncine, Bornia e Abbas (2012), reforçam que o custo por procedimento médico hospitalar fornece, dentre outros, alguns indicadores, tais como o procedimento com maior margem de contribuição, e o ponto de equilíbrio por procedimento, que é uma importante ferramenta de gestão de resultados operacionais. Porém, reforçam os referidos autores que, apesar do hospital obter informações mais adequadas para o gerenciamento da informação, não se verifica, na prática, a utilização da informação desta forma, havendo uma lacuna a ser preenchida pelo cálculo do custo por procedimento médico hospitalar.

O custo por procedimento compreende todos os insumos utilizados pelo paciente no fluxo percorrido ao longo das unidades funcionais do hospital, ou seja, um paciente que se submeteu ao procedimento cirurgia plástica de mamoplastia, receberá em sua conta custos da unidade de internação, custos do centro cirúrgico, da farmácia, enfim, todos os insumos e serviços que foram aplicados durante sua permanência no hospital.

Assim, tendo em vista a importância da beleza para a sociedade; a escassez de pesquisas relacionadas ao custo dos procedimentos estéticos, que segundo Avelar (2011) é alto; e a importância da obtenção do custo por procedimento (MATTOS, 2002; LEONCINE, 2010), o objetivo deste trabalho é calcular o custo dos três procedimentos cirúrgicos estéticos mais procurados pelos brasileiros.

Este artigo está dividido, além desta introdução, em mais três seções. A segunda seção apresenta a Fundamentação Teórica, em que incialmente discorre brevemente os aspectos introdutórios em relação aos procedimentos cirúrgicos para em seguida expor como obter o custo por procedimento. A terceira seção apresenta a metodologia, expondo os tipos de pesquisa (quanto aos objetivos, a natureza do problema, a abordagem do problema e aos procedimentos técnicos) utilizados. A quarta seção apresenta o cálculo dos custos das cirurgias plásticas, ou seja, o custo por procedimento. E por fim, a quinta seção apresenta as considerações finais do estudo.

\section{Fundamentação teórica}

\section{Cirurgia plástica}

Os hospitais, que durante o mercantilismo foram criados para o controle de doenças transmissíveis (ROSEN, 1958), a partir do final do século XVIII, devido ao avanço no diagnóstico e tratamento, passaram a apresentar um perfil de alta complexidade (ESTEVES, 1992).

Segundo Almeida (1983) um hospital é uma instituição destinada ao diagnóstico e tratamento de doentes; à prevenção de doenças; à prática, a pesquisa e ao ensino da medicina e da cirurgia, da enfermagem e das demais especialidades afins. 

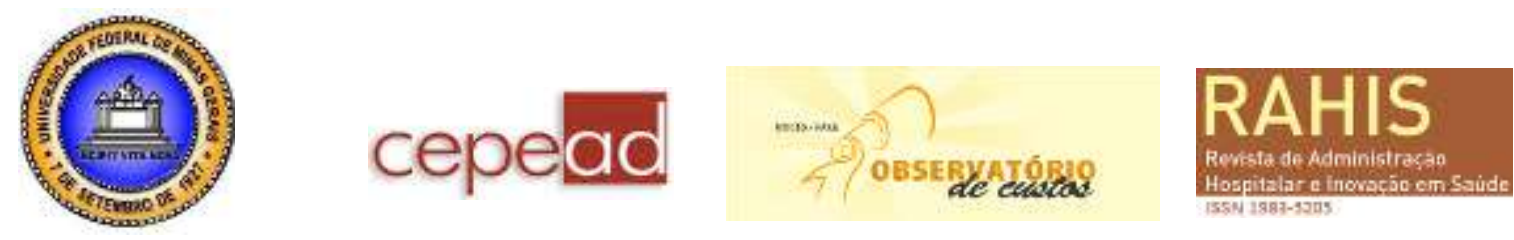

Entretanto, observa-se nas últimas décadas um crescimento marcante da beleza, no qual a busca da estética provoca uma série de novos consumos de bens e serviços, como exercícios, tratamentos específicos e a cirurgia plástica (BORELLI; CASOTTI, 2010).

Assim, no Brasil, como em outros países, as cirurgias plásticas passaram a fazer parte do cotidiano dos hospitais, que segundo Senhoras (2007) muitas das inovações tecnológicas ocorridas no setor, resultam na introdução de um novo serviço.

Existem relatos de que há quatro mil anos a.C., os hindus já realizavam técnicas para correções corporais. Também os egípcios, há menos de dois mil anos a.C., também utilizavam técnicas para amenizar defeitos, deformidades ou desfiguramentos provados por questões religiosas. Apenas a partir do século XIX que a especialidade passou a ser reconhecida como importante ramo da cirurgia (CURI, 2005 apud SANTE, 2008).

Segundo a Sociedade Brasileira de Cirurgia Plástica (SBPC, 2013), a cirurgia plástica, que pode ser reparadora ou estética, inclui os procedimentos cirúrgicos e também não cirúrgicos para remodelar as estruturas normais do corpo, com o objetivo de melhorar a aparência e autoestima do paciente.

Em relação a cirurgia plástica reparadora, Avelar (2000) e Medeiros (2004) citam que compreende as deformidades físicas devido a algum tipo de trauma facial (acidente de automóvel, queimadura, amputação, mordida de animal, de ser humano, etc.), ou devido à ressecção (remoção de tecido), de tumor (câncer), com destruição parcial ou total de lábio, nariz, pálpebras e orelhas. Já, a cirurgia plástica estética é utilizada, não para corrigir alguma imperfeição, mas sim, para melhorar a aparência e a autoestima.

Aquino (2009, p. 10), referindo-se ao conceito de cirurgia plástica estética, salienta que a intervenção "responde ao imediatismo da modernidade, trazendo resultados em curto prazo". Assim, o corpo tornou-se um importante capital (físico, simbólico, econômico e social) e o culto ao corpo se tornou um estilo de vida (Goldenberg, 2002; 2007 apud AQUINO, 2009).

Realizar uma cirurgia plástica estética ou reparadora é um acontecimento importante na vida, tanto das mulheres quanto dos homens. Askegaard, Gertsen e Langer (2002) mencionam que o bem estar, a autoestima e a autoconfiança são os motivos que levam as mulheres e homens a procurar técnicas para correções corporais. Insatisfação com a aparência, atitude defensiva e sensibilidade afetiva, são os motivos citados por Sante (2008) na busca da modificação corporal e facial.

Assim, diante desta realidade de busca da beleza ou da perfeição, e também dos gastos crescentes na área de saúde, conhecer e gerenciar os custos é necessário para se obter informações a serem utilizadas pelos gestores no controle e tomada de decisão. Mais especificamente, segundo Crispim (2010, p.27), o "conhecimento acerca do valor dos procedimentos médicos" é um diferencial competitivo. E, nas palavras de Fukumoto e Freitas (2000, p. 1), a "necessidade de conhecer melhor seus custos cresce, à medida que a concorrência aumenta e que o país se desenvolve".

\section{Custo por procedimento}

O crescimento e competição entre as entidades hospitalares torna imperativo uma utilização adequada de instrumentos de gestão de custos para a tomada de decisão dessas entidades.

Quando se tem informações detalhadas dos custos hospitalares, obtém-se um maior esclarecimento sobre o comportamento e as variáveis que influenciam os diversos serviços 

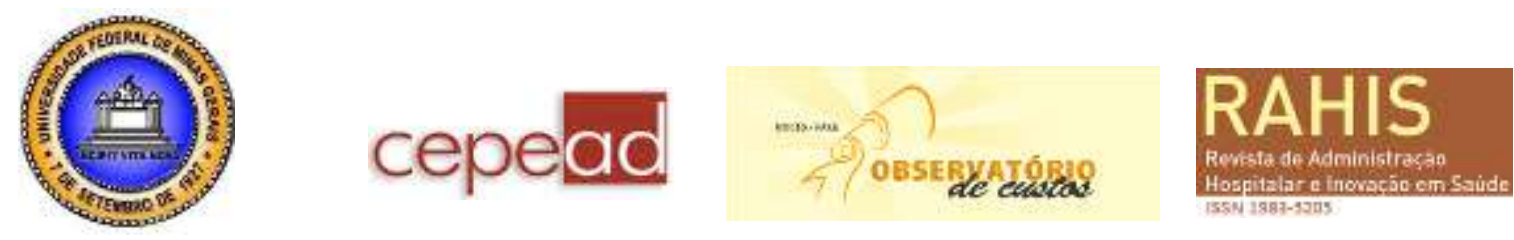

prestados pela entidade, permitindo assim, um gerenciamento dos custos por nível departamental, por procedimentos hospitalares específicos e mesmo por prestador ou provedor de serviço (STEPHENSON, 1985; TOSO, 1989; HILL; JOHNS, 1994 apud FALK, 2001).

Leoncine, Bornia e Abbas (2012) ressaltam que a apuração dos custos por procedimento médico-hospitalar contribui para a melhoria do gerenciamento operacional de instituições de saúde.

A definição do custeio por procedimentos hospitalares significa a constituição de custo sob a unidade do "paciente", ou seja, são alocados todos os insumos utilizados no fluxo percorrido pelo paciente, nos serviços prestados pelo hospital (MATOS, 2002).

Para Martins (2002, p. 82) "uma correta determinação do custo por procedimento médico é importante, porque afeta um grande número de ações do hospital". Segundo o referido autor, a determinação de custos por procedimento, proporciona os seguintes benefícios: i) estabelecer os preços de cada procedimento médico; ii) estimar os custos para negociação de pacotes; iii) estimar a rentabilidade das especialidades e procedimentos médicos; iv) determinar as margens brutas associadas às especialidades e procedimentos médicos; e v) confrontar o faturamento e custos do procedimento médico.

Para determinação dos custos por procedimentos hospitalares Leoncine, Bornia e Abbas (2012), apresentam em seu estudo uma sistemática para apuração de custos por procedimento médico-hospitalar. Em síntese, segundo esta sistemática, é preciso identificar os custos diretos e também os custos advindos dos centros de custos do hospital. Para o cálculo dos centros de custos, o método mais utilizado e adequado à realidade e complexidade dos hospitais, conforme Leoncine (2010), é o método das seções homogêneas (RKW).

No entanto, vários autores como, por exemplo, Baumgartner (1998), Vargas (2002), Schultz (2007), Paixão, Souza e Lima (2010), e Drumond et al. (2012) consideram o método do custeio baseado em atividades (ABC) como o mais adequado para hospitais. Leoncine (2010, p. 66) salienta que:

apesar do grande número de pesquisas envolvendo o $\mathrm{ABC}$ no ambiente hospitalar, empiricamente averigua-se que os hospitais brasileiros utilizam o RKW. O método é de valia para algumas áreas que desejam melhorar seu processo ou como cada atividade contribui para a melhoria de determinado procedimento médico.

Neste contexto, Abbas et al. (2012) mencionam que, apesar de vários autores defenderem a utilização do $\mathrm{ABC}$ em hospitais, a literatura apresenta a aplicação do método em apenas algumas áreas do hospital. Não há trabalhos que apresentam a aplicação do método no hospital como um todo, pois há uma complexidade envolvida nos mesmos. Segundo Bittencourt (1999), estas instituições agrupam várias funções, algumas com alto nível de especialização, outras com alta escala de produção, e também atividades administrativas.

A operacionalização do RKW, segundo Bornia (2010), é sintetizada em cinco fases:

i. separação dos custos e despesas em itens;

ii. divisão da empresa em centros de custos produtivos e auxiliares. Os centros produtivos, no caso dos hospitais, segundo Rocchi (1982) são os que prestam atendimentos diretamente aos pacientes; enquanto os centros auxiliares são os que prestam serviços ou dão apoio aos centros de custos produtivos, não atuando portanto diretamente sobre o paciente;

iii. distribuição por meio de rateios dos itens de custos e despesas aos centros de custos; 
iv. redistribuição dos custos dos centros auxiliares (administração, departamento pessoal, diretoria, contabilidade, faturamento, almoxarifado, compras, farmácia, lavanderia e rouparia, central de esterilização, higiene e limpeza, recepção, governança e, dentre outros) aos próprios auxiliares e também aos produtivos (centro cirúrgico, unidade de internação, ambulatório, tomografia, radiologia e pronto-socorro, dentre outros);

v. distribuição dos custos dos centros produtivos aos produtos, ou seja, no caso do hospitais, os custos são alocados ao custo do procedimento.

Quanto aos materiais e medicamentos, que são custos diretos, ou seja, custos em que é possível quantificar seu consumo no atendimento ao paciente de forma fácil e confiável sem recorrer a rateios, são lançados pela farmácia e pelo almoxarifado diretamente no custo por procedimento.

O custo por procedimento recebe, portanto, os custos incorridos nos centros de custos, que podem ser obtidos por meio do método RKW, e também os custos diretos, ou seja, os materiais e medicamentos consumidos durante o tratamento dos pacientes, conforme figura 1.

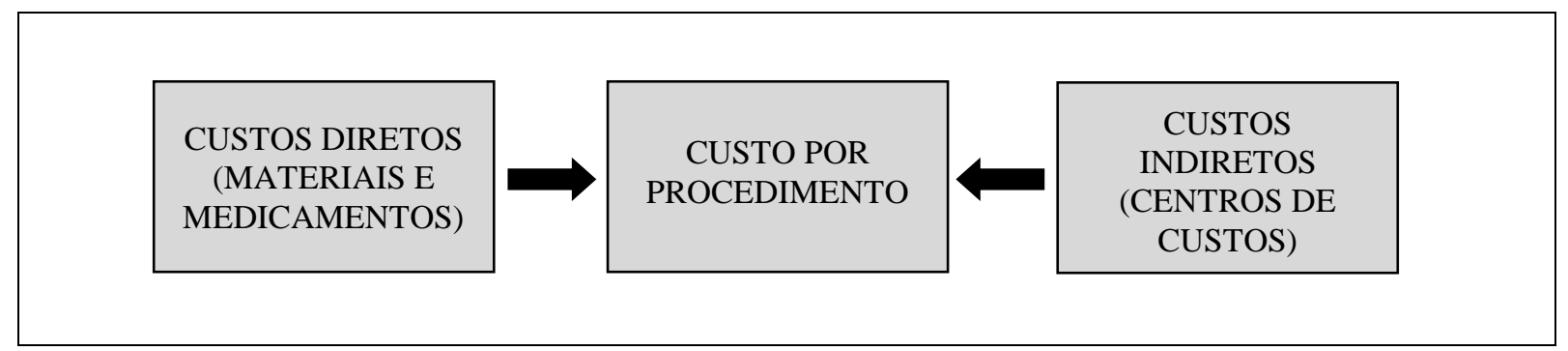

Fonte: Elaborado pelas autoras

Figura 1 - Custo por procedimento ressalta que:

Em relação ao método RKW e ao custeio por procedimentos, Matos (2002, p. 97-98)

a apropriação de custos por centros de custos e o custeio por procedimentos hospitalares representam as duas formas mais usuais de expressão do custo de um serviço gerado por uma empresa hospitalar. O custo gerado pela apropriação dos centros de custos corresponde às unidades de serviços produzidas em cada um dos centros de custos definidos para o hospital. As expressões de custo unitário associadas a cada um dos centros de custos corresponderão, portanto, a uma diária hospitalar, a uma taxa de sala cirúrgica, a uma consulta, a um exame, entre outros. O custo dos procedimentos hospitalares corresponde a uma sequência de cálculos, os quais compreenderão os custos unitários gerados pelos centros de custos combinados com a intensidade dos referidos insumos.

Segundo Martins (2000), para cada procedimento médico prepara-se uma ficha de custo. E, os cálculos apresentados nesta ficha de custos devem ser aprovados pela comissão ou a equipe de trabalho do hospital (MARTINS, 2000; LEONCINE; BORNIA; ABBAS, 2012).

O cálculo do custo de procedimentos hospitalares pode ser aplicado sob diferentes parâmetros de referência, que geralmente pode ser influenciado pelos objetivos da adoção desta metodologia, pelas condições de gestão existentes na instituição, e também os padrões das organizações das informações estatísticas e de custos (MATOS, 2002). 

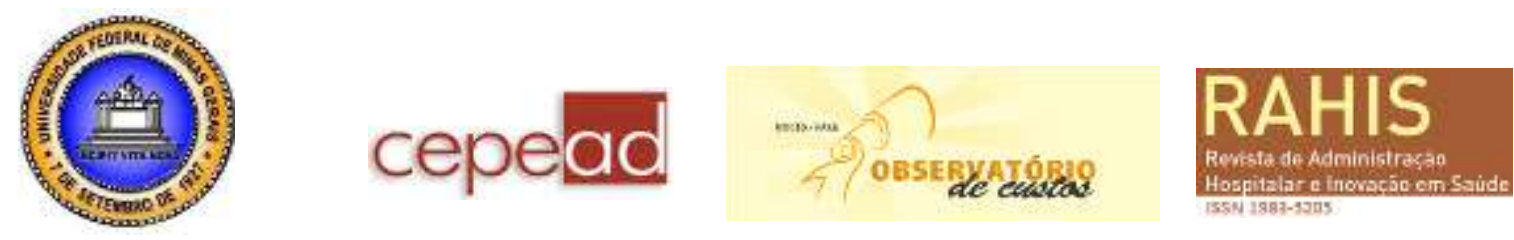

O custo de procedimentos hospitalares por paciente corresponde à demonstração analítica de todos os insumos consumidos pelo paciente, ou seja, a apropriação dos custos é específica a cada paciente atendido. O custo médio de procedimentos hospitalares é obtido pelas amostras aleatórias de pacientes que permitem um grau de representatividade adequado. E por fim, os custos de procedimentos hospitalares baseado em protocolos padrões de tratamentos, se baseiam na conduta médica adequada para o tratamento do paciente (MATOS, 2002).

\section{Metodologia}

Para Gil (2002) os aspectos metodológicos descrevem os procedimentos que serão utilizados na realização da pesquisa. Assim, de acordo com a classificação de Silva e Menezes (2007) esta pesquisa é descritiva, aplicada, quantitativa, bibliográfica e estudo de caso.

De acordo com os objetivos deste trabalho, esta pesquisa é classificada como descritiva, que segundo Beuren (2003, p. 81) "configura-se como um estudo intermediário entre a pesquisa exploratória e explicativa". Para Gil (2002) o objetivo deste tipo de pesquisa é descrever as características de determinada população ou estabelecer relações entre variáveis.

Do ponto de vista da sua natureza, esta pesquisa é aplicada, pois segundo Silva e Menezes (2007) tem como objetivo gerar conhecimentos para aplicação prática envolvidos na solução de problemas específicos. Segundo as referidas autoras, "envolve verdades e interesses locais" (SILVA; MENEZES, 2007, p. 20).

Quanto à abordagem do problema, ou seja, para a interpretação, descrição e explicação dos dados coletados, foi utilizada a pesquisa quantitativa, que, segundo Silva e Menezes (2001), traduz em números as informações para analisá-las.

E, quanto aos procedimentos técnicos é bibliografia e estudo de caso. A pesquisa bibliografia é o meio pelo qual se busca o domínio do estado da arte sobre determinado assunto, ou seja, "explica um problema a partir de referenciais teóricos publicados em documentos" (CERVO; BERVIAN, 1983, p. 55). Andrade (2003, p. 126) ressalta que "todo trabalho científico pressupõe uma pesquisa bibliográfica preliminar".

E, o estudo de caso, segundo Cervo e Bervian (1983) é o método que possibilita analisar um determinado grupo para examinar os aspectos envolvidos em torno deles. Ou seja, propiciam ao pesquisados a possibilidade de estudar dois ou mais sujeitos (TRIVIÑOS, 1987 apud BEUREN, 2003).

\section{Resultados}

\section{As cirurgias plásticas mais procuradas pelos brasileiros}

A lipoaspiração ocupa o primeiro lugar do ranking, em cirurgias estéticas mais procuradas pelos brasileiros, e consiste em remodelar áreas específicas do corpo, como barriga, coxas, braços, cintura, costas, pescoço, bochechas, pernas, dentre outros, por meio da remoção de depósitos de gordura.

A mamoplastia de aumento, que aparece em segundo lugar, se caracteriza pela inserção de prótese para aumento do volume da mama. O objetivo é tornar o corpo mais proporcional, ou mudar o formato e recuperar a forma devido à amamentação, ou ainda, igualar mamas assimétricas. 
E, a abdominoplastia, que aparece em terceiro lugar, consiste na remoção do excesso de gordura e de flacidez do abdômen com o objetivo de criar um perfil abdominal tonificado.

Em 2011, foram realizados 211.108 cirurgias de lipoaspiração, 148.962 cirurgias de mamoplastia e 95.004 de abdominoplastia, conforme tabela 1. Os dados referentes ao ano de 2012 ainda não foram apresentados pelo ISAPS.

Tabela 1 - Ranking das cirurgias plásticas mais procuradas pelos brasileiros

\begin{tabular}{lcc}
\hline Tipos de Cirurgias & Quantidade & \% \\
\hline Lipoaspiração & 211.108 & $46 \%$ \\
Mamoplastia & 148.962 & $33 \%$ \\
Abdominoplastia & 95.004 & $21 \%$ \\
TOTAL & $\mathbf{4 5 5 . 0 7 4}$ & $\mathbf{1 0 0 \%}$ \\
\hline
\end{tabular}

Fonte: Elaborado pelas autoras com base no ISAPS (2013)

Os três procedimentos devem obrigatoriamente ser realizados em hospitais, visto que, é necessária uma estrutura complexa composta por acomodações, centro cirúrgico, unidade de terapia intensiva, e profissionais especializados, tais como enfermeiros, médicos cirurgiões e anestesistas.

\section{Cálculo dos custos}

A coleta de dados ocorreu nos meses de maio, junho e julho de 2013 em um hospital de grande porte localizado no Estado do Paraná. As informações para o cálculo dos custos dos procedimentos foram extraídas do setor de custos do hospital, que não incorre no cálculo dos custos por procedimentos. Apenas separam os custos e despesas por itens e os distribuem aos centros de custos auxiliares e produtivos, ou seja, utilizam apenas as três primeiras fases do método RKW apresentadas por Bornia (2010) e que estão sintetizadas na fundamentação teórica. Portanto, a redistribuição dos custos dos centros auxiliares aos próprios auxiliares e também aos produtivos não é feita, bem como, não distribuem os custos dos centros produtivos aos procedimentos. Consequentemente, o hospital não conhece os custos dos seus procedimentos.

Para o cálculo do custo de procedimentos hospitalares foi aplicado o custo médio de procedimentos hospitalares, visto que, conforme apresentado por Matos (2002) na Fundamentação Teórica, as amostras aleatórias de pacientes permitem um grau adequado de representatividade, ou seja, não há heterogeneidade nos procedimentos cirúrgicos lipoaspiração, mamoplastia de aumento e abdominoplastia.

Em relação ao processo cirúrgico como um todo, após aprovação do médico para a realização do procedimento inicia-se o processo da cirurgia eletiva (programada com antecedência). O paciente é recepcionado no hospital, e em seguida o enfermeiro responsável admite-o para os procedimentos pré-operatórios e encaminha para o centro cirúrgico onde é realizado o procedimento. Encerrado o procedimento o paciente segue para a sala de Recuperação Pós-Anestésica (RPA) e desta para a Unidade de Internação onde receberá os cuidados necessários pelos enfermeiros até a liberação feita médico.

Para o cálculo do custo dos três procedimentos - lipoaspiração, mamoplastia de aumento e abdominoplastia - incialmente foram levantados os custos diretos. A tabela 2 

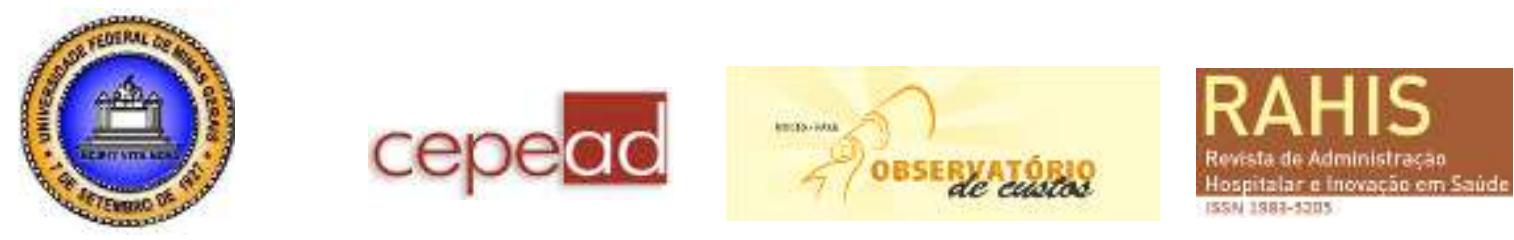

apresenta o consumo médio dos materiais, medicamentos, órteses e próteses e materiais especiais de cada procedimento cirúrgico.

Os materiais referem-se a agulhas, fios cirúrgicos, cânulas, compressas, luvas cirúrgicas, seringas e lâminas, dentre outros, utilizados em cada procedimento. Em relação aos medicamentos, referem-se ao soro, antitérmicos, anestésicos, antibióticos e antiinflamatórios usados para evitar dores, inflamações e efeitos colaterais que podem ser decorrentes do procedimento.

No caso da mamoplastia, as órteses e próteses e materiais especiais (OPME) apresentam alto custo. $\mathrm{O}$ valor incluso na tabela 2 , é referente a prótese mamária, que neste caso representa um custo médio, tendo em vista que existem especificações diferenciadas por paciente (modelos, tamanhos e marcas).

Tabela 2 - Custos diretos consumidos nos procedimentos

\begin{tabular}{lccc}
\hline \multicolumn{1}{c}{ Custos Diretos } & Lipoaspiração & Mamoplastia & Abdominoplastia \\
\hline Materiais & $\mathrm{R} \$ 350,58$ & $\mathrm{R} \$ 520,29$ & $\mathrm{R} \$ 348,20$ \\
Medicamentos & $\mathrm{R} \$ 199,20$ & $\mathrm{R} \$ 281,47$ & $\mathrm{R} \$ 127,92$ \\
OPME & $\mathrm{R} \$ 69,90$ & $\mathrm{R} \$ 1.801,90$ & $\mathrm{R} \$ 69,90$ \\
Custo Total & $\mathrm{R} \$ \mathbf{6 1 9 , 6 8}$ & $\mathrm{R} \$ \mathbf{2 . 6 0 3 , 6 6}$ & $\mathrm{R} \$ \mathbf{5 4 6 , 0 2}$ \\
\hline
\end{tabular}

Fonte: Elaborado pelas autoras

Após a identificação dos custos diretos, foram levantados também os custos dos centros de custos, que, conforme citado na fundamentação teórica, são obtidos por meio do método RKW. O quadro 1 apresenta os custos dos centros produtivos pelos quais cada procedimento passa, ou seja, são os centros que prestam atendimentos diretamente aos pacientes. Ressalta-se que os custos dos centros auxiliares já foram distribuídos aos centros produtivos.

\begin{tabular}{|c|c|c|}
\hline Tipos de Cirurgias & Unidade de Internação & Centro Cirúrgico \\
\hline Lipoaspiração & $\bullet$ & $\bullet$ \\
\hline Mamoplastia & $\bullet$ & $\bullet$ \\
\hline Abdominoplastia & $\bullet$ & $\bullet$ \\
\hline
\end{tabular}

Elaborado pelas autoras

Quadro 1 - Custo dos procedimentos

As tabelas 3, 4 e 5 apresentam os custos dos procedimentos, já inclusos os custos diretos (materiais, medicamentos, e órteses e próteses e materiais especiais) e os custos dos centros produtivos (Unidade de Internação e Centro Cirúrgico).

Os centros de custos produtivos receberam os custos dos próprios centros, como mão de obra direta, mão de obra indireta, depreciação de equipamentos, energia elétrica e aluguel, dentre outros, e também os custos dos centros auxiliares, como manutenção, lavanderia e os centros administrativos, dentre outros. Todos os centros produtivos possuem uma unidade de produção, no caso da Unidade de Internação, a unidade de produção é a diária. No caso do Centro Cirúrgico, a unidade de produção é a taxa de sala cirúrgica e também a taxa de sala de recuperação pós-anestésica. 
As tabelas não apresentam os valores dos honorários médicos, visto que, não representam um custo do hospital. No caso dos três procedimentos em análise, tanto o médico cirurgião plástico quanto o médico anestesista são remunerados pelos próprios pacientes. Além disso, os valores dos honorários médicos são diferenciados. A Fundação Instituto de Pesquisas Econômicas da Universidade de São Paulo (FIPE), apoiada pelas entidades médicas nacionais, estabelece um padrão mínimo e ético para remuneração dos procedimentos médicos, que leva em consideração a complexidade técnica, o tempo de execução do ato médico, a atenção requerida e o grau de treinamento dos médicos.

O procedimento cirúrgico lipoaspiração apresenta um custo de $\mathrm{R} \$ 1.347,38$, sendo que os custos com material direto são responsáveis por $46 \%$ dos custos, enquanto os custos dos centros produtivos correspondem por $54 \%$ dos custos totais (Tabela 3 ). 
Tabela 3 - Custo do procedimento lipoaspiração

\begin{tabular}{|c|c|c|c|c|}
\hline \multicolumn{5}{|c|}{ CUSTO POR PROCEDIMENTO: LIPOASPIRAÇÃO } \\
\hline Descrição & $\begin{array}{l}\text { Unidade de } \\
\text { Mensuração }\end{array}$ & Quantidade & $\begin{array}{c}\text { Custo } \\
\text { Unitário }\end{array}$ & Custo Total \\
\hline 1. Materiais e Medicamentos: & & & & \\
\hline - Canula... & unidade & 1,00 & 2,44 & 2, \\
\hline • Fio de sutura........................ & envelope & 4,00 & 17,81 & 71,24 \\
\hline . & unidade & 1,00 & 1,52 & 1,52 \\
\hline - Soro fisiologico .. & frasco & 2,00 & 1,88 & 3,76 \\
\hline
\end{tabular}

$\Lambda \Lambda \Lambda \Lambda \Lambda \Lambda \Lambda \Lambda \Lambda \Lambda \Lambda \Lambda \Lambda \Lambda \Lambda \Lambda \Lambda \Lambda \Lambda \Lambda \Lambda \Lambda \Lambda \Lambda \Lambda \Lambda \Lambda \Lambda \Lambda \Lambda \Lambda \Lambda \Lambda \Lambda \Lambda \Lambda \Lambda \Lambda \Lambda \Lambda \Lambda \Lambda \Lambda \Lambda \Lambda \Lambda \Lambda \Lambda \Lambda \Lambda \Lambda \Lambda \Lambda \Lambda \Lambda \Lambda \Lambda \Lambda \Lambda \Lambda \Lambda \Lambda \Lambda \Lambda \Lambda \Lambda \Lambda \Lambda \Lambda \Lambda \Lambda \Lambda \Lambda \Lambda \Lambda \Lambda \Lambda \Lambda \Lambda \Lambda \Lambda \Lambda \Lambda \Lambda \Lambda$ $\Lambda \Lambda \Lambda \Lambda \Lambda \Lambda \Lambda \Lambda \Lambda \Lambda \Lambda \Lambda \Lambda \Lambda \Lambda \Lambda \Lambda \Lambda \Lambda \Lambda \Lambda \Lambda \Lambda \Lambda \Lambda \Lambda \Lambda \Lambda \Lambda \Lambda \Lambda \Lambda \Lambda \Lambda \Lambda \Lambda \Lambda \Lambda \Lambda \Lambda \Lambda \Lambda \Lambda \Lambda \Lambda \Lambda \Lambda \Lambda \Lambda \Lambda \Lambda \Lambda \Lambda \Lambda \Lambda \Lambda \Lambda \Lambda \Lambda \Lambda \Lambda \Lambda \Lambda \Lambda \Lambda \Lambda \Lambda \Lambda \Lambda \Lambda \Lambda \Lambda \Lambda \Lambda \Lambda \Lambda \Lambda \Lambda \Lambda \Lambda \Lambda \Lambda \Lambda \Lambda \Lambda$

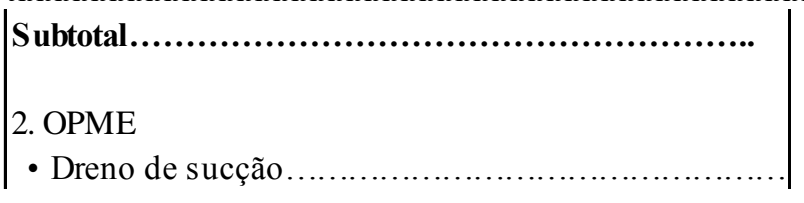
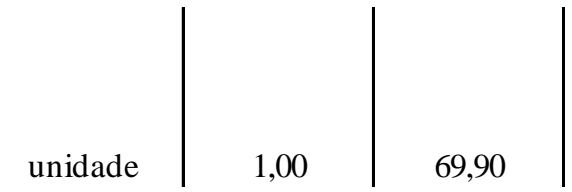

549,78

$\Lambda \Lambda \Lambda \Lambda \Lambda \Lambda \Lambda \Lambda \Lambda \Lambda \Lambda \Lambda \Lambda \Lambda \Lambda \Lambda \Lambda \Lambda \Lambda \Lambda \Lambda \Lambda \Lambda \Lambda \Lambda \Lambda \Lambda \Lambda \Lambda \Lambda \Lambda \Lambda \Lambda \Lambda \Lambda \Lambda \Lambda \Lambda \Lambda \Lambda \Lambda \Lambda \Lambda \Lambda \Lambda \Lambda \Lambda \Lambda \Lambda \Lambda \Lambda \Lambda \Lambda \Lambda \Lambda \Lambda \Lambda \Lambda \Lambda \Lambda \Lambda \Lambda \Lambda \Lambda \Lambda \Lambda \Lambda \Lambda \Lambda \Lambda \Lambda \Lambda \Lambda \Lambda \Lambda \Lambda \Lambda \Lambda \Lambda \Lambda \Lambda \Lambda \Lambda \Lambda \Lambda$ $\Lambda \Lambda \Lambda \Lambda \Lambda \Lambda \Lambda \Lambda \Lambda \Lambda \Lambda \Lambda \Lambda \Lambda \Lambda \Lambda \Lambda \Lambda \Lambda \Lambda \Lambda \Lambda \Lambda \Lambda \Lambda \Lambda \Lambda \Lambda \Lambda \Lambda \Lambda \Lambda \Lambda \Lambda \Lambda \Lambda \Lambda \Lambda \Lambda \Lambda \Lambda \Lambda \Lambda \Lambda \Lambda \Lambda \Lambda \Lambda \Lambda \Lambda \Lambda \Lambda \Lambda \Lambda \Lambda \Lambda \Lambda \Lambda \Lambda \Lambda \Lambda \Lambda \Lambda \Lambda \Lambda \Lambda \Lambda \Lambda \Lambda \Lambda \Lambda \Lambda \Lambda \Lambda \Lambda \Lambda \Lambda \Lambda \Lambda \Lambda \Lambda \Lambda \Lambda \Lambda \Lambda$
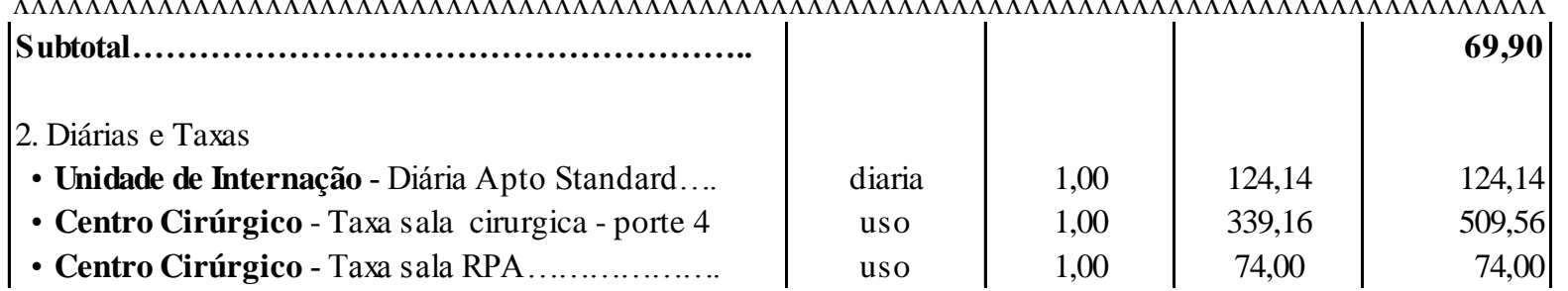

$\Lambda \Lambda \Lambda \Lambda \Lambda \Lambda \Lambda \Lambda \Lambda \Lambda \Lambda \Lambda \Lambda \Lambda \Lambda \Lambda \Lambda \Lambda \Lambda \Lambda \Lambda \Lambda \Lambda \Lambda \Lambda \Lambda \Lambda \Lambda \Lambda \Lambda \Lambda \Lambda \Lambda \Lambda \Lambda \Lambda \Lambda \Lambda \Lambda \Lambda \Lambda \Lambda \Lambda \Lambda \Lambda \Lambda \Lambda \Lambda \Lambda \Lambda \Lambda \Lambda \Lambda \Lambda \Lambda \Lambda \Lambda \Lambda \Lambda \Lambda \Lambda \Lambda \Lambda \Lambda \Lambda \Lambda \Lambda \Lambda \Lambda \Lambda \Lambda \Lambda \Lambda \Lambda \Lambda \Lambda \Lambda \Lambda \Lambda \Lambda \Lambda \Lambda \Lambda \Lambda \Lambda$ $\Lambda \Lambda \Lambda \Lambda \Lambda \Lambda \Lambda \Lambda \Lambda \Lambda \Lambda \Lambda \Lambda \Lambda \Lambda \Lambda \Lambda \Lambda \Lambda \Lambda \Lambda \Lambda \Lambda \Lambda \Lambda \Lambda \Lambda \Lambda \Lambda \Lambda \Lambda \Lambda \Lambda \Lambda \Lambda \Lambda \Lambda \Lambda \Lambda \Lambda \Lambda \Lambda \Lambda \Lambda \Lambda \Lambda \Lambda \Lambda \Lambda \Lambda \Lambda \Lambda \Lambda \Lambda \Lambda \Lambda \Lambda \Lambda \Lambda \Lambda \Lambda \Lambda \Lambda \Lambda \Lambda \Lambda \Lambda \Lambda \Lambda \Lambda \Lambda \Lambda \Lambda \Lambda \Lambda \Lambda \Lambda \Lambda \Lambda \Lambda \Lambda \Lambda \Lambda \Lambda \Lambda$

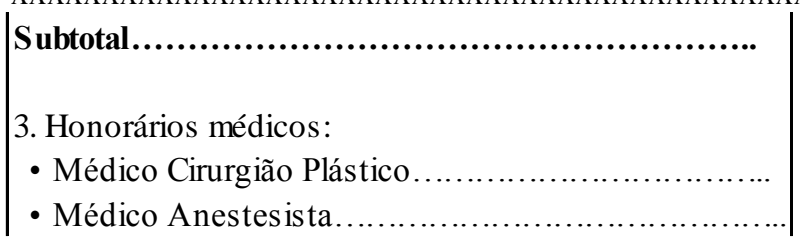
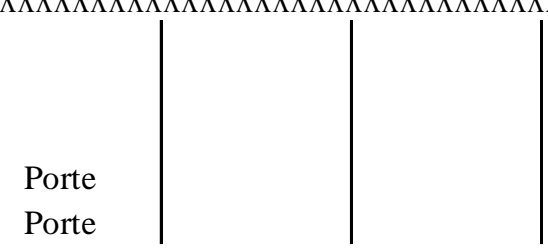

727,70

$\Lambda \Lambda \Lambda \Lambda \Lambda \Lambda \Lambda \Lambda \Lambda \Lambda \Lambda \Lambda \Lambda \Lambda \Lambda \Lambda \Lambda \Lambda \Lambda \Lambda \Lambda \Lambda \Lambda \Lambda \Lambda \Lambda \Lambda \Lambda \Lambda \Lambda \Lambda \Lambda \Lambda \Lambda \Lambda \Lambda \Lambda \Lambda \Lambda \Lambda \Lambda \Lambda \Lambda \Lambda \Lambda \Lambda \Lambda \Lambda \Lambda \Lambda \Lambda \Lambda \Lambda \Lambda \Lambda \Lambda \Lambda \Lambda \Lambda \Lambda \Lambda \Lambda \Lambda \Lambda \Lambda \Lambda \Lambda \Lambda \Lambda \Lambda \Lambda \Lambda \Lambda \Lambda \Lambda \Lambda \Lambda \Lambda \Lambda \Lambda \Lambda \Lambda \Lambda \Lambda \Lambda$ $\Lambda \Lambda \Lambda \Lambda \Lambda \Lambda \Lambda \Lambda \Lambda \Lambda \Lambda \Lambda \Lambda \Lambda \Lambda \Lambda \Lambda \Lambda \Lambda \Lambda \Lambda \Lambda \Lambda \Lambda \Lambda \Lambda \Lambda \Lambda \Lambda \Lambda \Lambda \Lambda \Lambda \Lambda \Lambda \Lambda \Lambda \Lambda \Lambda \Lambda \Lambda \Lambda \Lambda \Lambda \Lambda \Lambda \Lambda \Lambda \Lambda \Lambda \Lambda \Lambda \Lambda \Lambda \Lambda \Lambda \Lambda \Lambda \Lambda \Lambda \Lambda \Lambda \Lambda \Lambda \Lambda \Lambda \Lambda \Lambda \Lambda \Lambda \Lambda \Lambda \Lambda \Lambda \Lambda \Lambda \Lambda \Lambda \Lambda \Lambda \Lambda \Lambda \Lambda \Lambda \Lambda$

\begin{tabular}{|l|l|l|r|} 
Subtotal................................................ & & & $\mathbf{0 , 0 0}$ \\
\hline Total & & & \\
\end{tabular}

Fonte: Elaborado pelas autoras

O procedimento cirúrgico mamoplastia apresenta um custo de $\mathrm{R} \$ 3.457,50$, sendo que os custos com material direto são responsáveis por $75 \%$ dos custos, enquanto os custos dos centros produtivos correspondem por $25 \%$ dos custos totais. O alto percentual de custos diretos se deve à prótese mamária (Tabela 4). 


\section{cepead}

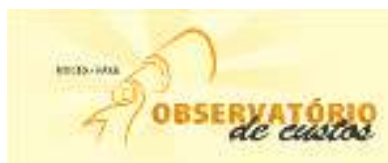

Tabela 4 - Custo do procedimento mamoplastia

\begin{tabular}{|c|c|c|c|c|}
\hline \multicolumn{5}{|c|}{ CUSTO POR PROCEDIMENTO: MAMOPLASTIA } \\
\hline Descrição & $\begin{array}{l}\text { Unidade de } \\
\text { Mensuração }\end{array}$ & Quantidade & $\begin{array}{c}\text { Custo } \\
\text { Unitário }\end{array}$ & Custo Total \\
\hline 1. Materiais e Medicamentos: & & & & \\
\hline - Compressa gaze.......... & unidade & 5,00 & 3,30 & \\
\hline - Fio de sutura... & envelope & 10,00 & 14,35 & 143,50 \\
\hline - Seringa perfusora.. & unidade & 1,00 & 6,94 & 6, \\
\hline - Plasil.. & unidade & 1,00 & 0,96 & 0,96 \\
\hline - Outros mat/med & diversos & 1,00 & 633,86 & 633,86 \\
\hline
\end{tabular}

$\Lambda \Lambda \Lambda \Lambda \Lambda \Lambda \Lambda \Lambda \Lambda \Lambda \Lambda \Lambda \Lambda \Lambda \Lambda \Lambda \Lambda \Lambda \Lambda \Lambda \Lambda \Lambda \Lambda \Lambda \Lambda \Lambda \Lambda \Lambda \Lambda \Lambda \Lambda \Lambda \Lambda \Lambda \Lambda \Lambda \Lambda \Lambda \Lambda \Lambda \Lambda \Lambda \Lambda \Lambda \Lambda \Lambda \Lambda \Lambda \Lambda \Lambda \Lambda \Lambda \Lambda \Lambda \Lambda \Lambda \Lambda \Lambda \Lambda \Lambda \Lambda \Lambda \Lambda \Lambda \Lambda \Lambda \Lambda \Lambda \Lambda \Lambda \Lambda \Lambda \Lambda \Lambda \Lambda \Lambda \Lambda \Lambda \Lambda \Lambda \Lambda \Lambda \Lambda \Lambda \Lambda$ $\Lambda \Lambda \Lambda \Lambda \Lambda \Lambda \Lambda \Lambda \Lambda \Lambda \Lambda \Lambda \Lambda \Lambda \Lambda \Lambda \Lambda \Lambda \Lambda \Lambda \Lambda \Lambda \Lambda \Lambda \Lambda \Lambda \Lambda \Lambda \Lambda \Lambda \Lambda \Lambda \Lambda \Lambda \Lambda \Lambda \Lambda \Lambda \Lambda \Lambda \Lambda \Lambda \Lambda \Lambda \Lambda \Lambda \Lambda \Lambda \Lambda \Lambda \Lambda \Lambda \Lambda \Lambda \Lambda \Lambda \Lambda \Lambda \Lambda \Lambda \Lambda \Lambda \Lambda \Lambda \Lambda \Lambda \Lambda \Lambda \Lambda \Lambda \Lambda \Lambda \Lambda \Lambda \Lambda \Lambda \Lambda \Lambda \Lambda \Lambda \Lambda \Lambda \Lambda \Lambda \Lambda$
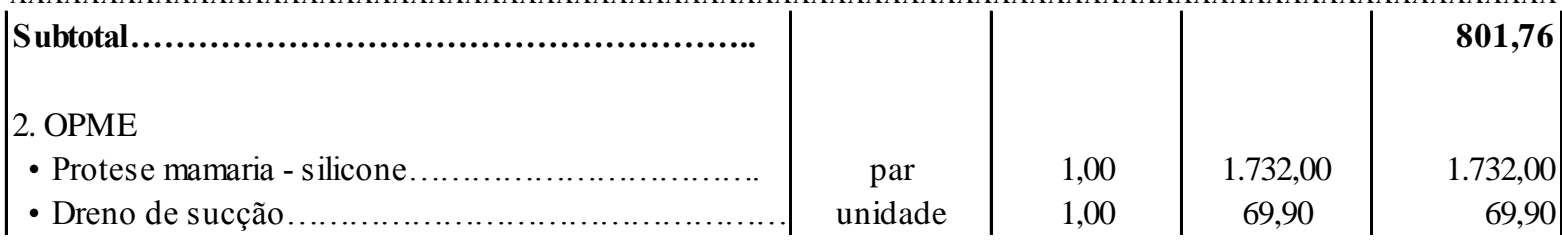

$\Lambda \Lambda \Lambda \Lambda \Lambda \Lambda \Lambda \Lambda \Lambda \Lambda \Lambda \Lambda \Lambda \Lambda \Lambda \Lambda \Lambda \Lambda \Lambda \Lambda \Lambda \Lambda \Lambda \Lambda \Lambda \Lambda \Lambda \Lambda \Lambda \Lambda \Lambda \Lambda \Lambda \Lambda \Lambda \Lambda \Lambda \Lambda \Lambda \Lambda \Lambda \Lambda \Lambda \Lambda \Lambda \Lambda \Lambda \Lambda \Lambda \Lambda \Lambda \Lambda \Lambda \Lambda \Lambda \Lambda \Lambda \Lambda \Lambda \Lambda \Lambda \Lambda \Lambda \Lambda \Lambda \Lambda \Lambda \Lambda \Lambda \Lambda \Lambda \Lambda \Lambda \Lambda \Lambda \Lambda \Lambda \Lambda \Lambda \Lambda \Lambda \Lambda \Lambda \Lambda \Lambda$ $\Lambda \Lambda \Lambda \Lambda \Lambda \Lambda \Lambda \Lambda \Lambda \Lambda \Lambda \Lambda \Lambda \Lambda \Lambda \Lambda \Lambda \Lambda \Lambda \Lambda \Lambda \Lambda \Lambda \Lambda \Lambda \Lambda \Lambda \Lambda \Lambda \Lambda \Lambda \Lambda \Lambda \Lambda \Lambda \Lambda \Lambda \Lambda \Lambda \Lambda \Lambda \Lambda \Lambda \Lambda \Lambda \Lambda \Lambda \Lambda \Lambda \Lambda \Lambda \Lambda \Lambda \Lambda \Lambda \Lambda \Lambda \Lambda \Lambda \Lambda \Lambda \Lambda \Lambda \Lambda \Lambda \Lambda \Lambda \Lambda \Lambda \Lambda \Lambda \Lambda \Lambda \Lambda \Lambda \Lambda \Lambda \Lambda \Lambda \Lambda \Lambda \Lambda \Lambda \Lambda \Lambda$ Subtotal.

3. Diárias e Taxas

- Unidade de Internação - Diária Apto Standard....

- Centro Cirúrgico - Taxa sala cirurgica - porte 5

- Centro Cirúrgico - Taxa sala RPA.

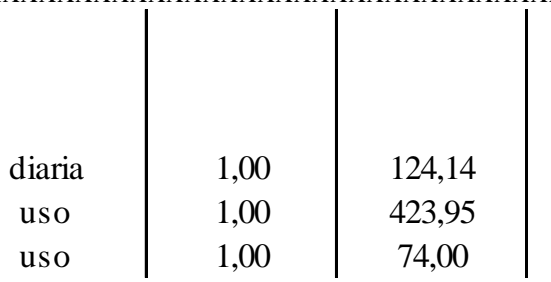

$1.801,90$ (⿸丆口

Subtotal.

4. Honorários médicos:

- Médico Cirurgião Plástico

- Médico Anestesista.

Porte

Porte

$\Lambda \Lambda \Lambda \Lambda \Lambda \Lambda \Lambda \Lambda \Lambda \Lambda \Lambda \Lambda \Lambda \Lambda \Lambda \Lambda \Lambda \Lambda \Lambda \Lambda \Lambda \Lambda \Lambda \Lambda \Lambda \Lambda \Lambda \Lambda \Lambda \Lambda \Lambda \Lambda \Lambda \Lambda \Lambda \Lambda \Lambda \Lambda \Lambda \Lambda \Lambda \Lambda \Lambda \Lambda \Lambda \Lambda \Lambda \Lambda \Lambda \Lambda \Lambda \Lambda \Lambda \Lambda \Lambda \Lambda \Lambda \Lambda \Lambda \Lambda \Lambda \Lambda \Lambda \Lambda \Lambda \Lambda \Lambda \Lambda \Lambda \Lambda \Lambda \Lambda \Lambda \Lambda \Lambda \Lambda \Lambda \Lambda \Lambda \Lambda \Lambda \Lambda \Lambda \Lambda \Lambda$ $\Lambda \Lambda \Lambda \Lambda \Lambda \Lambda \Lambda \Lambda \Lambda \Lambda \Lambda \Lambda \Lambda \Lambda \Lambda \Lambda \Lambda \Lambda \Lambda \Lambda \Lambda \Lambda \Lambda \Lambda \Lambda \Lambda \Lambda \Lambda \Lambda \Lambda \Lambda \Lambda \Lambda \Lambda \Lambda \Lambda \Lambda \Lambda \Lambda \Lambda \Lambda \Lambda \Lambda \Lambda \Lambda \Lambda \Lambda \Lambda \Lambda \Lambda \Lambda \Lambda \Lambda \Lambda \Lambda \Lambda \Lambda \Lambda \Lambda \Lambda \Lambda \Lambda \Lambda \Lambda \Lambda \Lambda \Lambda \Lambda \Lambda \Lambda \Lambda \Lambda \Lambda \Lambda \Lambda \Lambda \Lambda \Lambda \Lambda \Lambda \Lambda \Lambda \Lambda \Lambda \Lambda$

\begin{tabular}{|l|l|r|r|r|} 
Subtotal................................................ & & & & $\mathbf{0 , 0 0}$ \\
\hline Total & & & & $\mathbf{3 . 4 5 7 , 5 0}$ \\
\hline
\end{tabular}

Fonte: Elaborado pelas autoras

O procedimento cirúrgico abdominoplastia apresenta um custo de $\mathrm{R} \$ 1.371,11$, sendo que os custos com material direto são responsáveis por $40 \%$ dos custos, enquanto os custos dos centros produtivos correspondem por $60 \%$ dos custos totais (Tabela 5).

A tabela 6 apresenta o percentual de custos dos procedimentos. Em relação aos materiais diretos, o procedimento cirúrgico mamoplastia apresenta o percentual mais alto, devido à prótese mamária que faz com que o custo desta cirurgia seja mais alto em relação às demais.

Assim, comparando os três procedimentos, constata-se que os custos que mais se diferenciam dizem respeito a prótese mamária. Portanto, os custos dos medicamentos, diárias e taxas é muito parecido para os três procedimentos, bem como, os custos dos centros produtivos ( $\mathrm{R} \$ 727,70 ; \mathrm{R} \$ 853,84 ; \mathrm{R} \$ 825,09)$. 
Tabela 5 - Custo do procedimento abdominoplastia

\begin{tabular}{|c|c|c|c|c|}
\hline \multicolumn{5}{|c|}{ CUSTO POR PROCEDIMENTO: ABDOMINOPLASTIA } \\
\hline Descrição & $\begin{array}{l}\text { Unidade de } \\
\text { Mensuração }\end{array}$ & Quantidade & $\begin{array}{c}\text { Custo } \\
\text { Unitário }\end{array}$ & Custo Total \\
\hline 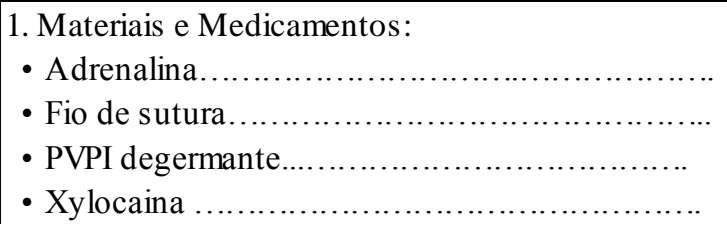 & $\begin{array}{l}\text { ampola } \\
\text { envelope } \\
\text { ml } \\
\text { ampola }\end{array}$ & $\begin{array}{c}4,00 \\
10,00 \\
100,00 \\
1,00\end{array}$ & $\begin{array}{c}0,96 \\
17,41 \\
0,02 \\
1,57\end{array}$ & $\begin{array}{r}3,84 \\
174,10 \\
2,00 \\
1,57\end{array}$ \\
\hline
\end{tabular}

$\Lambda \Lambda \Lambda \Lambda \Lambda \Lambda \Lambda \Lambda \Lambda \Lambda \Lambda \Lambda \Lambda \Lambda \Lambda \Lambda \Lambda \Lambda \Lambda \Lambda \Lambda \Lambda \Lambda \Lambda \Lambda \Lambda \Lambda \Lambda \Lambda \Lambda \Lambda \Lambda \Lambda \Lambda \Lambda \Lambda \Lambda \Lambda \Lambda \Lambda \Lambda \Lambda \Lambda \Lambda \Lambda \Lambda \Lambda \Lambda \Lambda \Lambda \Lambda \Lambda \Lambda \Lambda \Lambda \Lambda \Lambda \Lambda \Lambda \Lambda \Lambda \Lambda \Lambda \Lambda \Lambda \Lambda \Lambda \Lambda \Lambda \Lambda \Lambda \Lambda \Lambda \Lambda \Lambda \Lambda \Lambda \Lambda \Lambda \Lambda \Lambda \Lambda \Lambda \Lambda \Lambda$ $\Lambda \Lambda \Lambda \Lambda \Lambda \Lambda \Lambda \Lambda \Lambda \Lambda \Lambda \Lambda \Lambda \Lambda \Lambda \Lambda \Lambda \Lambda \Lambda \Lambda \Lambda \Lambda \Lambda \Lambda \Lambda \Lambda \Lambda \Lambda \Lambda \Lambda \Lambda \Lambda \Lambda \Lambda \Lambda \Lambda \Lambda \Lambda \Lambda \Lambda \Lambda \Lambda \Lambda \Lambda \Lambda \Lambda \Lambda \Lambda \Lambda \Lambda \Lambda \Lambda \Lambda \Lambda \Lambda \Lambda \Lambda \Lambda \Lambda \Lambda \Lambda \Lambda \Lambda \Lambda \Lambda \Lambda \Lambda \Lambda \Lambda \Lambda \Lambda \Lambda \Lambda \Lambda \Lambda \Lambda \Lambda \Lambda \Lambda \Lambda \Lambda \Lambda \Lambda \Lambda \Lambda$

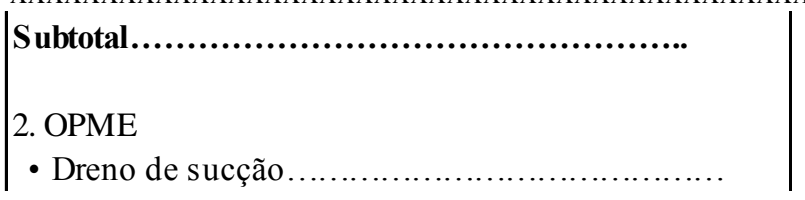
unidade

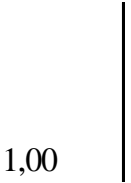

$\Lambda \Lambda \Lambda \Lambda \Lambda \Lambda \Lambda \Lambda \Lambda \Lambda \Lambda \Lambda \Lambda \Lambda \Lambda \Lambda \Lambda \Lambda \Lambda \Lambda \Lambda \Lambda \Lambda \Lambda \Lambda \Lambda \Lambda \Lambda \Lambda \Lambda \Lambda \Lambda \Lambda \Lambda \Lambda \Lambda \Lambda \Lambda \Lambda \Lambda \Lambda \Lambda \Lambda \Lambda \Lambda \Lambda \Lambda \Lambda \Lambda \Lambda \Lambda \Lambda \Lambda \Lambda \Lambda \Lambda \Lambda \Lambda \Lambda \Lambda \Lambda \Lambda \Lambda \Lambda \Lambda \Lambda \Lambda \Lambda \Lambda \Lambda \Lambda \Lambda \Lambda \Lambda \Lambda \Lambda \Lambda \Lambda \Lambda \Lambda \Lambda \Lambda \Lambda \Lambda \Lambda$ $\Lambda \Lambda \Lambda \Lambda \Lambda \Lambda \Lambda \Lambda \Lambda \Lambda \Lambda \Lambda \Lambda \Lambda \Lambda \Lambda \Lambda \Lambda \Lambda \Lambda \Lambda \Lambda \Lambda \Lambda \Lambda \Lambda \Lambda \Lambda \Lambda \Lambda \Lambda \Lambda \Lambda \Lambda \Lambda \Lambda \Lambda \Lambda \Lambda \Lambda \Lambda \Lambda \Lambda \Lambda \Lambda \Lambda \Lambda \Lambda \Lambda \Lambda \Lambda \Lambda \Lambda \Lambda \Lambda \Lambda \Lambda \Lambda \Lambda \Lambda \Lambda \Lambda \Lambda \Lambda \Lambda \Lambda \Lambda \Lambda \Lambda \Lambda \Lambda \Lambda \Lambda \Lambda \Lambda \Lambda \Lambda \Lambda \Lambda \Lambda \Lambda \Lambda \Lambda \Lambda \Lambda$
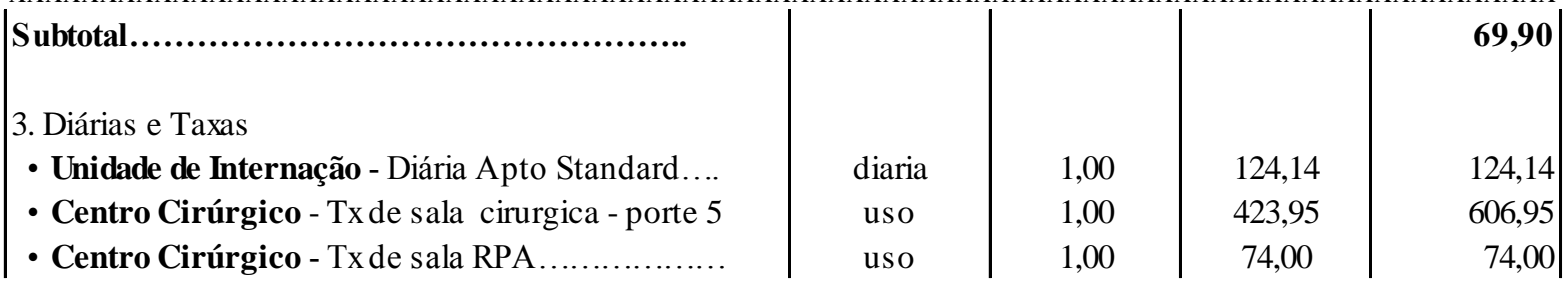

$\Lambda \Lambda \Lambda \Lambda \Lambda \Lambda \Lambda \Lambda \Lambda \Lambda \Lambda \Lambda \Lambda \Lambda \Lambda \Lambda \Lambda \Lambda \Lambda \Lambda \Lambda \Lambda \Lambda \Lambda \Lambda \Lambda \Lambda \Lambda \Lambda \Lambda \Lambda \Lambda \Lambda \Lambda \Lambda \Lambda \Lambda \Lambda \Lambda \Lambda \Lambda \Lambda \Lambda \Lambda \Lambda \Lambda \Lambda \Lambda \Lambda \Lambda \Lambda \Lambda \Lambda \Lambda \Lambda \Lambda \Lambda \Lambda \Lambda \Lambda \Lambda \Lambda \Lambda \Lambda \Lambda \Lambda \Lambda \Lambda \Lambda \Lambda \Lambda \Lambda \Lambda \Lambda \Lambda \Lambda \Lambda \Lambda \Lambda \Lambda \Lambda \Lambda \Lambda \Lambda \Lambda$ $\Lambda \Lambda \Lambda \Lambda \Lambda \Lambda \Lambda \Lambda \Lambda \Lambda \Lambda \Lambda \Lambda \Lambda \Lambda \Lambda \Lambda \Lambda \Lambda \Lambda \Lambda \Lambda \Lambda \Lambda \Lambda \Lambda \Lambda \Lambda \Lambda \Lambda \Lambda \Lambda \Lambda \Lambda \Lambda \Lambda \Lambda \Lambda \Lambda \Lambda \Lambda \Lambda \Lambda \Lambda \Lambda \Lambda \Lambda \Lambda \Lambda \Lambda \Lambda \Lambda \Lambda \Lambda \Lambda \Lambda \Lambda \Lambda \Lambda \Lambda \Lambda \Lambda \Lambda \Lambda \Lambda \Lambda \Lambda \Lambda \Lambda \Lambda \Lambda \Lambda \Lambda \Lambda \Lambda \Lambda \Lambda \Lambda \Lambda \Lambda \Lambda \Lambda \Lambda \Lambda \Lambda$ Subtotal.

4. Honorários médicos:

- Médico Cirurgião Plástico

- Médico Anestesista.

Porte

$\Lambda \Lambda \Lambda \Lambda \Lambda \Lambda \Lambda \Lambda \Lambda \Lambda \Lambda \Lambda \Lambda \Lambda \Lambda \Lambda \Lambda \Lambda \Lambda \Lambda \Lambda \Lambda \Lambda \Lambda \Lambda \Lambda \Lambda \Lambda \Lambda \Lambda \Lambda \Lambda \Lambda \Lambda \Lambda \Lambda \Lambda \Lambda \Lambda \Lambda \Lambda \Lambda \Lambda \Lambda \Lambda \Lambda \Lambda \Lambda \Lambda \Lambda \Lambda \Lambda \Lambda \Lambda \Lambda \Lambda \Lambda \Lambda \Lambda \Lambda \Lambda \Lambda \Lambda \Lambda \Lambda \Lambda \Lambda \Lambda \Lambda \Lambda \Lambda \Lambda \Lambda \Lambda \Lambda \Lambda \Lambda \Lambda \Lambda \Lambda \Lambda \Lambda \Lambda \Lambda \Lambda$ $\Lambda \Lambda \Lambda \Lambda \Lambda \Lambda \Lambda \Lambda \Lambda \Lambda \Lambda \Lambda \Lambda \Lambda \Lambda \Lambda \Lambda \Lambda \Lambda \Lambda \Lambda \Lambda \Lambda \Lambda \Lambda \Lambda \Lambda \Lambda \Lambda \Lambda \Lambda \Lambda \Lambda \Lambda \Lambda \Lambda \Lambda \Lambda \Lambda \Lambda \Lambda \Lambda \Lambda \Lambda \Lambda \Lambda \Lambda \Lambda \Lambda \Lambda \Lambda \Lambda \Lambda \Lambda \Lambda \Lambda \Lambda \Lambda \Lambda \Lambda \Lambda \Lambda \Lambda \Lambda \Lambda \Lambda \Lambda \Lambda \Lambda \Lambda \Lambda \Lambda \Lambda \Lambda \Lambda \Lambda \Lambda \Lambda \Lambda \Lambda \Lambda \Lambda \Lambda \Lambda \Lambda$

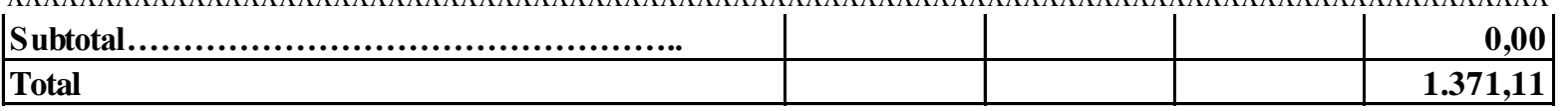

Fonte: Elaborado pelas autoras

Tabela 6 - Comparação dos custos dos procedimentos

\begin{tabular}{cccc}
\hline Custos Diretos & Lipoaspiração & Mamoplastia & Abdominoplastia \\
\hline Materiais Diretos & $46 \%$ & $75 \%$ & $40 \%$ \\
Centros de Custos Produtivos & $54 \%$ & $25 \%$ & $60 \%$ \\
Custo Total & $\mathbf{1 0 0 \%}$ & $\mathbf{1 0 0 \%}$ & $\mathbf{1 0 0 \%}$ \\
\hline
\end{tabular}

Fonte: Elaborado pelas autoras

Com esses valores é possível, dentre outros, aplicar o preço de venda dos procedimentos, negociar os preços com os convênios, controlar os custos, comparar os custos 

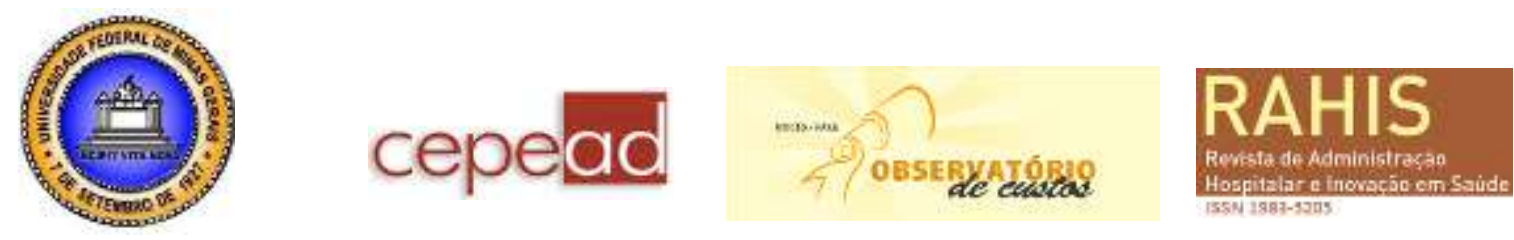

e preços com outros hospitais, e também verificar quais os procedimentos são mais viáveis para o hospital.

Entretanto, o preço de venda dos procedimentos nem sempre é um fator determinante para quem opta por realizar uma cirurgia plástica estética. A reputação e notoriedade do médico é um item forte que impacta na escolha. Portanto, é importante que o hospital seja atrativo e atenda às expectativas dos médicos.

Para o cálculo dos custos dos procedimentos, não foram computados os honorários médicos (cirurgião e anestesista), visto que, não fazem parte dos custos do hospital, ou seja, são remunerados pelos próprios pacientes e não pelo hospital.

Diferentemente das tabelas 3, 4 e 5, que apresentam o valor do custo dos procedimentos cirúrgicos estéticos para o hospital, a tabela 7 apresenta o preço final para o paciente.

Para a definição do índice do markup, o hospital considera que deve cobrir os custos diretos e indiretos (materiais e medicamentos; OPME; diárias e taxas), os impostos, os gastos não inclusos no custo, ou seja, as despesas administrativas, e o lucro desejado.

Ressalta-se porém, que no caso do procedimento mamoplastia, o markup não cobrirá o valor integral do item OPME, que neste caso refere-se ao valor pago pelas próteses mamárias ( $\mathrm{R} \$ 1.732,00)$. Logo, o indíce do markup incidirá sobre o valor de $\mathrm{R} \$ 1.725,50$, que representa o somatório dos materiais e medicamentos; OPME (apenas o dreno); e as diárias e taxas

O valor dos honorários médicos é relativo, ou seja, cada cirurgião pode estipular um valor que considera justo para seus honorários. Porém, dentre outros, a notoriedade do cirurgião e o grau de complexidade da cirurgia contribuem para um aumento nos valores. Assim, na tabela 7 este valor refere-se a uma média do preço dos procedimentos cirúrgicos estéticos mais procurados pelos brasileiros.

Tabela 7 - Preço final dos procedimentos

\begin{tabular}{lccr}
\hline & Lipoaspiração & Mamoplastia & Abdominoplastia \\
\hline Materiais e Medicamentos & $\mathrm{R} \$ 549,79$ & $\mathrm{R} \$ 801,76$ & $\mathrm{R} \$ 476,12$ \\
OPME & $\mathrm{R} \$ 69,90$ & $\mathrm{R} \$ 1.801,90$ & $\mathrm{R} \$ 69,90$ \\
Diárias e Taxas & $\mathrm{R} \$ 727,70$ & $\mathrm{R} \$ 853,84$ & $\mathrm{R} \$ 825,09$ \\
Total dos Custos hospitalares & $\mathrm{R} \$ 1.347,38$ & $\mathrm{R} \$ 3.457,50$ & $\mathrm{R} \$ 1.371,11$ \\
Markup & 1,64 & 1,33 & 1,75 \\
TOTAL & $\mathrm{R} \$ 2.200,00$ & $\mathrm{R} \$ 2.300,00$ & $\mathrm{R} \$ 2.400,00$ \\
Honorários Médicos & $\mathrm{R} \$ 4.500,00$ & $\mathrm{R} \$ 1.800,00$ & $\mathrm{R} \$ 4.000,00$ \\
Preço para o Paciente & $\mathbf{R} \mathbf{6 . 7 0 0 , 0 0}$ & $\mathbf{R} \mathbf{5 . 8 3 2 , 0 0}$ & $\mathbf{R} \mathbf{6 . 4 0 0 , 0 0}$ \\
\hline
\end{tabular}

Fonte: Elaborado pelas autoras

\section{Considerações finais}

Esta pesquisa teve como objetivo principal calcular o custo dos três procedimentos cirúrgicos estéticos mais procurados pelos brasileiros. Para tanto, baseado nas metodologias de Matos (2002) e Leoncine, Bornia e Abbas (2012), foram alocados aos procedimentos todos os insumos utilizados no fluxo percorrido pelo paciente nos serviços prestados pelo hospital, ou seja, tanto os custos diretos quanto os indiretos advindos da farmácia, do almoxarifado e dos centros de custos que foram obtidos por do método RKW. 

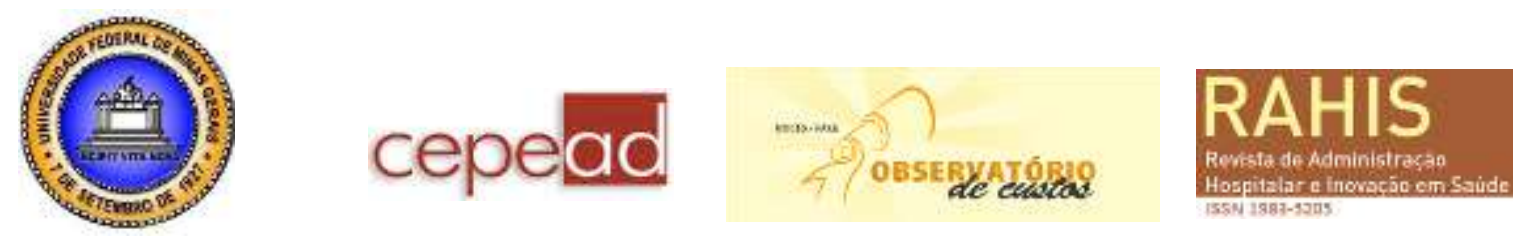

A pesquisa foi realizada em um hospital de grande porte, no período de maio, junho e julho de 2013 e constatou-se que não há diferenças significativas em relação aos custos dos materiais, medicamentos e dos centros produtivos para os três procedimentos. No entanto, o procedimento cirúrgico mamoplastia, apresentou um custo maior em relação à lipoaspiração e à abdominoplastia, devido ao alto custo da prótese mamária.

Vale ressaltar que, para o cálculo dos custos dos procedimentos, não foram computados os honorários médicos (cirurgião e anestesista), visto que, não fazem parte dos custos do hospital, ou seja, são remunerados pelos próprios pacientes.

Porém, tendo em vista que os leitores geralmente se sentem atraídos pela informação do valor que o paciente terá que dispender nos procedimentos, a tabela 7 apresenta o preço final para o paciente, ou seja, o valor que ele terá que desembolsar, considerando os materiais e medicamentos; OPME; diárias e taxas; e índice de markup, que devem ser pagos ao hospital, bem como os honorários médicos.

A literatura (STEPHENSON, 1985; TOSO, 1989; HILL; JOHNS, 1994 apud FALK, 2001; MATOS, 2002; LEONCINE, BORNIA E ABBAS, 2012) aponta que o cálculo dos custos dos procedimentos cirúrgicos é um fator importante para gerenciamento operacional e tomada de decisão dos gestores dos hospitais.

Apresenta também a literatura que a determinação de custos por procedimento facilita a análise de resultados do hospital, visto que, permite o agrupamento dos procedimentos hospitalares por procedimento cirúrgico; facilita o controle dos custos utilizados em cada procedimento; permite realizar comparações de custos, qualidade e preços com outras instituições e também estabelecer preços de venda sob a metodologia de pacotes para cada procedimento.

Assim, tendo em vista a importância da beleza para a sociedade e o grande número de procedimentos cirúrgicos estéticos realizados por ano, conhecer e os custos envolvidos com a indústria da beleza é um diferencial competitivo.

Estas conclusões devem ser consideradas apenas no contexto do universo, ou seja, no contexto do hospital em estudo. E como contribuição, o estudo exploratório apresenta indícios de que o custo por procedimento hospitalar é uma ferramenta essencial para quem quer se manter competitivo na área da saúde.

Para futuros trabalhos sugere-se realizar um estudo comparativo com outros hospitais que apurem os custos dos procedimentos, bem como calcular os custos de outros tipos de procedimentos para verificar quais são os mais rentáveis para o hospital.

\section{Referências}

ABBAS, K. Gestão de custos em organizações hospitalares. Dissertação (Mestrado em Engenharia de Produção) - Programa de Pós-Graduação em Engenharia de Produção, Universidade Federal de Santa Catarina, Florianópolis, 2001.

ABBAS, K. et al. Os métodos de custeio e sua aplicabilidade nos diversos tipos de organizações. In: CONGRESSO INTERNACIONAL DE ADMINISTRAÇÃO, 2012, Ponta Grossa, Anais...

ALMEIDA, T. R. R. Perspectivas de sobrevivência do hospital. Revista Paulista de Hospitais, São Paulo, n.5/6, p.104-113, maio/jun. 1983. 

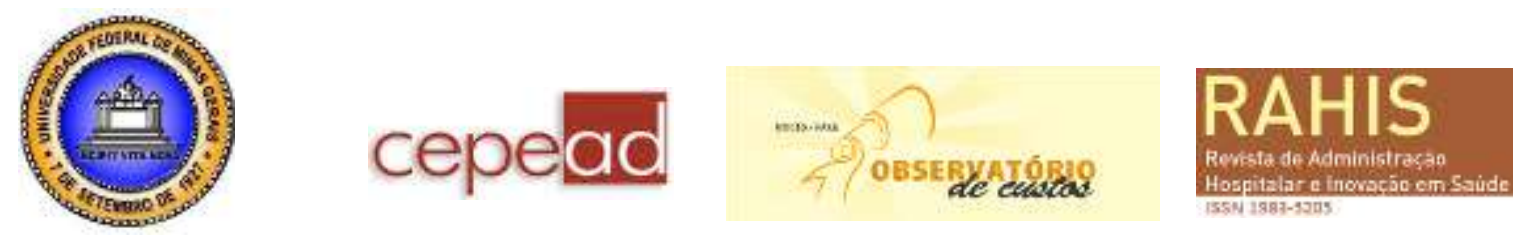

ANDRADE, M. M. Introdução à metodologia do trabalho científico. 6. Ed. São Paulo: Atlas, 2003.

AQUINO, T. A. M. Do "se esconder" ao "se mostrar": cirurgia plástica e normalização entre mulheres jovens de classe popular. Dissertação (Mestrado em Psicologia) - Programa de Pós-Graduação em Psicologia, Universidade Federal de Pernambuco, Recife, 2009.

ASKEGAARD, S.; GERTSEN, M. C.; LANGER, R. The body consumed: reflexivity and cosmetic surgery. Psychology \& Marketing, v. 19, n. 10, p. 793-812, 2002.

AVELAR, C. F. P. Personalidade e propensão à cirurgia plástica estética. Dissertação (Mestrado em Administração) - Centro de Pós-Graduação e Pesquisas em Administração. Departamento de Ciências Administrativas. Faculdade de Ciências Econômicas. Universidade Federal de Minas Gerais. Belo Horizonte, 2011.

AVELAR, J. M. Cirurgia Plástica: obrigação de meio e não obrigação de fim ou de resultado. São Paulo: Hipócrates, 2000.

BAUMGARTNER, R. R. Avaliação da aplicabilidade do custeio ABC - activity based costing na acurácia de custos na área hospitalar, especificamente na Unidade de Terapia Intensiva: estudo de um caso prático. Dissertação (Mestrado em Ciências Financeiras e Contábeis) - PUC, São Paulo, 1998.

BEUREN, I.M. Como elaborar trabalhos monográficos em contabilidade. In: LONGARAY, A. A.; RAUPP, F. M.; SOUSA, M. A. B.; COLAUTO, R. D.; PORTON, R. A. B.; BEUREN, I. M. (Org.). São Paulo: Atlas, 2003.

BITTENCOURT, O. N. S. O emprego do método de custeio baseado em atividades: Activity-based costing - como instrumento de apoio à decisão na área hospitalar. Dissertação (Mestrado em Administração) - Programa de Pós Graduação em Administração, Universidade Federal do Rio Grande do Sul, Porto Alegre, 1999.

BORELLI, F. C.; CASOTTI, L. M. O antes e o depois no consumo da cirurgia plástica: um estudo exploratório com jovens mulheres. In: ENCONTRO DE MARKETING DA ANPAD, IV, 2010, Florianópolis. Anais...

BORNIA, A. C. Análise gerencial de custos: aplicação em empresas modernas. 3. ed. São Paulo: Atlas, 2010.

CERVO; A. L.; BERVIAN, P. A. Metodologia da pesquisa: para uso dos estudantes universitários. São Paulo: McGraw-Hill do Brasil, 1983.

CRISPIM, C. H. Comportamento dos custos em hospitais administrados pela Secretaria de Estado da Saúde de Santa Catarina. Dissertação (Mestrado em Contabilidade) Programa de Pós-Graduação em Contabilidade, Universidade Federal de Santa Catarina, Florianópolis, 2010. 

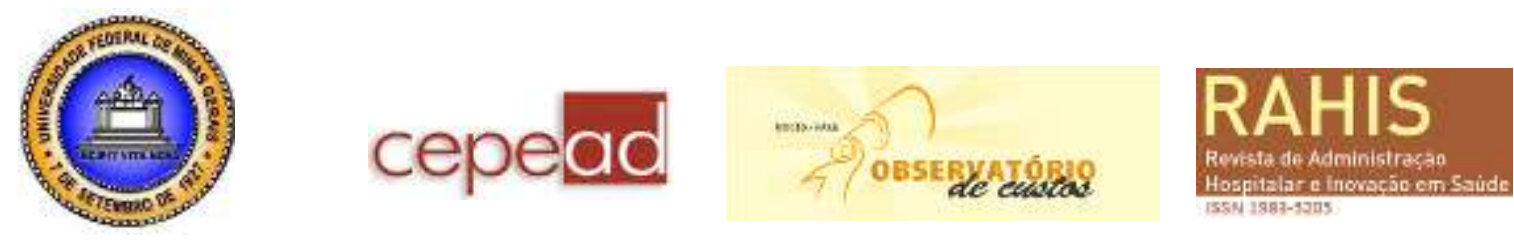

DRUMOND, H. A. et al. O uso do custeio baseado em atividades (ABC - activity based costing) na análise do processo de transplantes de fígado do Estado de Minas Gerais. In: CONGRESSO BRASILEIRO DE CUSTOS, VII, 2012, Bento Gonçalves, Anais...

ESTEVES, M. J. V. A utilização de custos em hospitais paulistas: um estudo preliminar. Dissertação (Mestrado em Saúde Pública) - Departamento de Prática de Saúde Pública. Faculdade de Saúde Pública da Universidade de São Paulo. São Paulo, 1992.

FALK, J. A. Gestão de custos para hospitais. São Paulo: Atlas, 2001.

FARIA, M. P. O corpo na mídia e o culto ao corpo na contemporaneidade. In: CONGRESSO DE CIÊNCIAS DA COMUNICAÇÃO NA REGIÃO CENTRO-OESTE, XII, 2010. Goiás, Anais...

FERRAZ, S. B.; SERRALTA, F. B. O impacto da cirurgia plástica na auto-estima. Estudos e Pesquisas em Psicologia. Rio de Janeiro, v. 7, n. 3, p. 557-569, dez. 2007.

FUKUMOTO, H. L.; FREITAS, R. Implantação do custeio baseado em atividades ABC/ABM no setor hospitalar: banco de sangue, um caso prático. In: CONGRESSO BRASILEIRO DE CUSTOS, VII, 2000, Recife, Anais...

GIL, A. C. Como elaborar projetos de pesquisa. 4. Ed. São Paulo: Atlas, 2002.

GOULART, R. L. Custeio baseado em atividade (ABC) aplicado em um serviço de radiologia em unidade hospitalar. Dissertação (Mestrado em Engenharia de Produção) Programa de Pós-Graduação em Engenharia de Produção, Universidade Federal de Santa Catarina, Florianópolis, 2000.

ISAPS - International Society of Aesthetic Plastic Surgeons. Disponível em: <http://www.isaps.org>. Acesso em: 12 jul. 2013.

LEONCINE, M. Sistemática para apuração de custos por procedimento médico hospitalar. Dissertação (Mestrado em Engenharia de Produção) - Programa de PósGraduação em Engenharia de Produção, Universidade Federal de Santa Catarina, Florianópolis, 2010.

LEONCINE, M.; BORNIA; A. C.; ABBAS, K. Sistemática para apuração de custos por procedimento médico hospitalar. Produção, São Paulo, v. 22, 2012.

MARTINS, D. Custos e orçamentos hospitalares. São Paulo: Atlas, 2000.

MARTINS, V. F. Desenvolvimento de um modelo de resultados em serviços hospitalares com base na comparação entre receitas e custos das atividades associadas aos serviços. Dissertação (Mestrado em Engenharia de Produção) - Programa de Pós-Graduação em Engenharia de Produção, Universidade Federal de Santa Catarina, Florianópolis, 2002. 

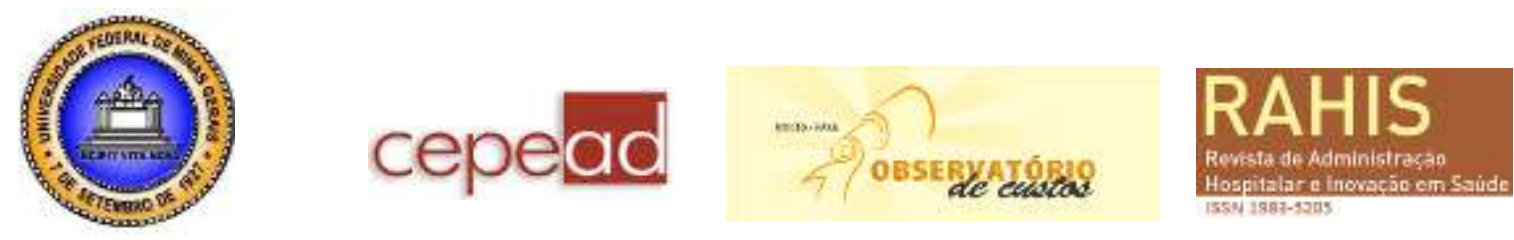

MATOS, A. J. Gestão de custos hospitalares: técnicas, análise e tomada de decisão. São Paulo: Editora STS, 2002.

MEDEIROS, M. S. F. Imagens, percepções e significados do corpo nas classes populares. Sociedade e estado. Brasília, v. 19, n. 2, 2004.

NERI, M. G. C. F. Aplicação do método de custeio abc na produção e utilização de hemocomponentes. Dissertação (Mestrado em Clínica Médica) - Programa de PósGraduação de Ciências Médicas, Universidade Estadual de Campinas, Campinas, 2006.

PAIXÃO, E. M.; SOUZA, A. A.; LIMA, L. C. M. Custo do parto normal e cesáreo: replicação do custeio ABC. In: CONGRESSO BRASILEIRO DE CUSTOS, XVII, 2010, Belo Horizonte, Anais...

ROCCHI, C. A. Apuração de custos em estabelecimentos hospitalares. Revista Brasileira de Contabilidade, Brasília, n. 41, p.19-27, 1982.

ROSEN, G. A history of public health. New York: M. D. Publications, 1958.

SANTE, A. B. Auto-imagem e características de personalidade na busca de cirurgia plástica estética. Dissertação (Mestrado em Psicologia) - Programa de Pós-Graduação em Psicologia. Faculdade de Filosofia, Ciências e Letras de Ribeirão Preto. Departamento de Psicologia e Educação, Universidade de São Paulo, Ribeirão Preto, São Paulo, 2008.

SBPC - SOCIEDADE BRASILEIRA DE CIRURGIA PLASTICA. Cirurgias e procedimentos. Disponível em: <http://www2.cirurgiaplastica.org.br/cirurgias-eprocedimentos/>. Acesso em: 17 jul. 2013.

SCHULTZ, C. A. Gestão dos custos de serviços públicos de alta complexidade hospitalar. Dissertação (Mestrado em Contabilidade) - Programa de Pós Graduação em Contabilidade, UFSC, Florianópolis, 2007.

SENHORAS, E. M. A cultura na organização hospitalar e as políticas culturais de coordenação de comunicação e aprendizagem. RECIIS - R. Eletr. de Com. Inov. Saúde. Rio de Janeiro, v. 1, n. 1, jan./jun. 2007.

SILVA, E. L.; MENEZES, E. M. Metodologia da pesquisa e elaboração de dissertação. 3. ed. Florianópolis: UFSC/PPGEP/LED, 2007.

SOUZA, A. A. et al. Modelagem do custeio baseado em atividades para o centro cirúrgico de hospitais. In: ENCONTRO NACIONAL DE ENGENHARIA DE PRODUÇÃO, XXVIII, 2008, Rio de Janeiro. Anais...

SOUZA, A. A. et al. Custos dos serviços hospitalares: análise da maternidade de um hospital da região metropolitana de Belo Horizonte, MG. In: CONGRESSO BRASILEIRO DE CUSTOS, XIX, 2012, Bento Gonçalves. Anais... 
VARGAS, O. C. O custeio baseado em atividades aplicado em serviços de UTI hospitalar. Dissertação (Mestrado em Engenharia de Produção) - Programa de PósGraduação em Engenharia de Produção, Universidade Federal de Santa Catarina, Florianópolis, 2002. 